\title{
Propriedades Termodinâmicas da Interação de Solventes Orgânicos com Fulerenos
}

Laryssa Missias de Faria $^{1 *}(\mathrm{PG})$, Luciano Ribeiro ${ }^{2}(\mathrm{PQ})$

laryssamissias3@gmail.com

${ }^{1}$ Universidade Estadual de Goiás, CCET. BR 153 Quadra Área km 99, Anápolis - GO. 75132-903

Palavras-chave: Fulereno, Constantes Espectroscópicas, Funções termodinâmicas

\section{Introdução}

Os fulerenos constituem uma classe de nanomoléculas formados por dezenas de átomos de carbono ${ }^{1}$. Suas aplicações vão desde a produção de cosméticos e medicamentos até a aplicação em células fotovoltaicas ${ }^{2}$. Diante da diversidade de suas aplicações esse trabalho buscou relacionar os cálculos das energias e constantes rovibracionais espectroscópicas com as propriedades termodinâmicas dos fulerenos em solventes orgânicos. Foram construídas curvas de energias potenciais (CEPs) para o sistema em questão. Com isso, buscou-se obter tanto os valores das propriedades espectroscópicas quanto termodinâmicas. Para a determinação das propriedades termodinâmicas foram utilizadas metodologias baseadas nos conceitos da mecânica quântica, considerando que o sistema formado pelo fulereno e o solvente se comporta como um sistema diatômico.

\section{Metodologia}

Os cálculos de estrutura eletrônica para a construção das curvas de energia potencial foi feito empregando-se o nível de teoria $\omega \mathrm{B} 9 \mathrm{XD} / 6-31 \mathrm{G}(\mathrm{d})$ no programa Gaussian 09. A obtenção das propriedades espectroscópicas foi realizado com o método da representação da variável discreta ${ }^{3}$ combinado com a utilização de equação da energia rovibracional diatômica e complementada pela utilização do método de Dunham ${ }^{4}$. As funções termodinâmicas foram obtidas através da função de partição rovibracional ${ }^{5}$.

\section{Resultados e Discussão}

Na Figura 1 são apresentadas as curvas de energia potencial da interação do fulereno $\mathrm{C}_{60}$ com os solventes mentano (símbolo em triângulo), hexano ao longo do diâmetro (símbolo em quadrado) e perpendicular ao diâmetro (símbolo em círculo). Pode-se notar, Figura 1 que a CEP para o hexano perpendicular ao diâmetro apresenta maior profundidade no poço, evidenciando que essa configuração é a mais indicada para o sistema em questão.

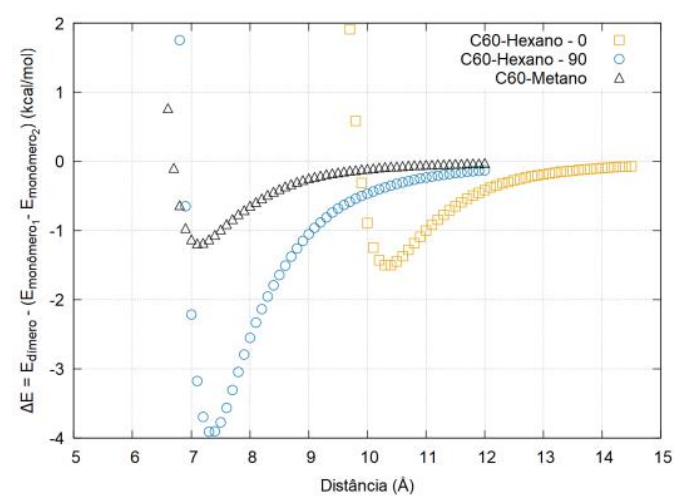

Figura 1. Curva de energia potencial C60-solventes orgânicos

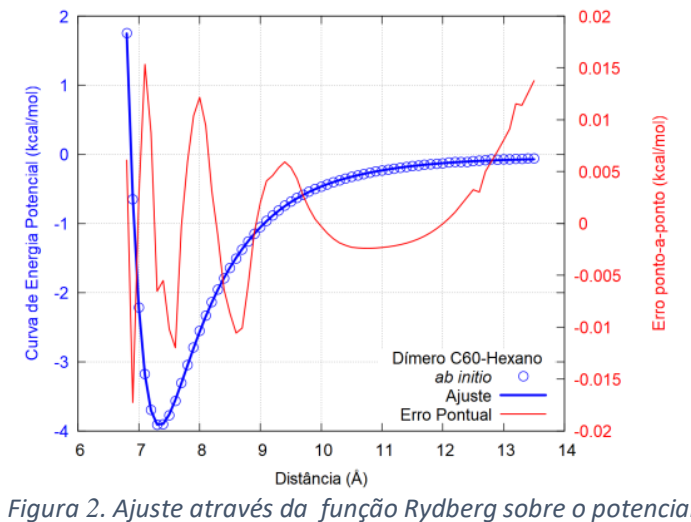

de interação do sistema.

A Figura 2 apresenta o ajuste realizado através da função analítica de Rydberg sobre o potencial de interação entre o fulereno e o solvente hexano perpendicular ao diâmetro, como está apresentado na Figura 1. Na Figura 2, a linha vermelha descreve o erro pontoa-ponto, a linha azul o ajuste realizado e os círculos abertos os pontos ab initio. Após o ajuste realizado, nota-se a formação de uma curva de energia potencial característica do sistema estudado. Na Tabela 1 são apresentados os valores das constantes espectroscópicas calculados pelos métodos de DVR e Dunhan. As constantes espectroscópicas associada a frequência de equilíbrio vibracional harmônica $\omega_{e}$ e a 
contribuição anarmônica $\omega_{e} x_{e}$ calculadas para os sistemas mostrados na Tabela 1, apresentam pequenas variações de um método para outro. Já rotacional de acoplamento $\alpha_{e}$ e a contribuição anarmônica $\omega_{e} y_{e}$ apresentam valores distintos. As propriedades termodinâmicas foram obtidas a partir dos valores das propriedades espectroscópicas apresentadas na Tabela 1.

Tabela 1. Valores das constantes espectroscópicas (em cm-1) calculadas pelos métodos de Dunham e DVR.

\begin{tabular}{ccccccc}
\hline Sistema & Métodos & $B e$ & $\omega_{e}$ & $\omega_{e} x_{e}$ & $\omega_{e} \mathrm{y}_{e}$ & $\alpha_{e}$ \\
\hline $\mathrm{C}_{60}$-Metano & Dunham & 0.0040 & 50.8 & 0.62 & 0.0137 & $2.10 .10^{-5}$ \\
& DVR & - & 50.8 & 0.62 & 0.0005 & -6.0971 \\
$\mathrm{C}_{60}$-Hexano & Dunham & 0.0211 & 63.7 & 3.03 & 0.5111 & $1.69 .10^{-6}$ \\
& DVR & - & 63.8 & 3.48 & 0.0665 & 0.0005 \\
\hline
\end{tabular}

Foram calculadas as funções que descrevem a variação da energia livre de Gibbs $\Delta \mathrm{G}$, a variação da entropia $\Delta \mathrm{S}$ e a variação da entalpia $\Delta \mathrm{H}$ e ainda a capacidade calorífica.

A Figura 3 apresenta a variação da entalpia para o sistema C60-Metano e C60-Hexano perpendicular ao eixo. Pode-se notar que a variação de entalpia segue o mesmo padrão observado na variação da energia de dissociação.

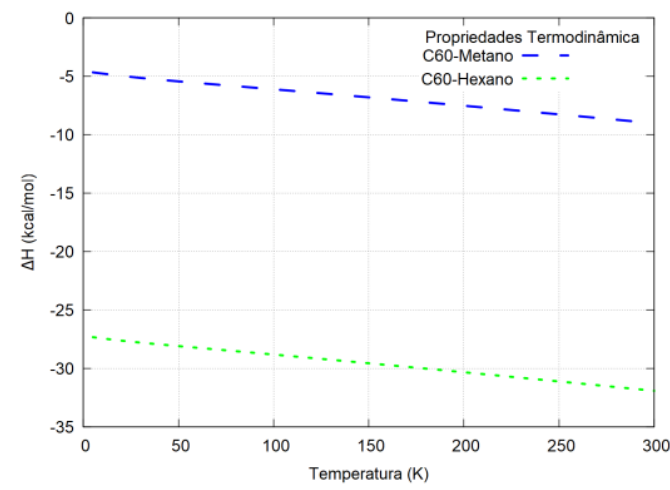

Figura 3. Variação da entalpia para o sistema C60-metano e C60-hexano.

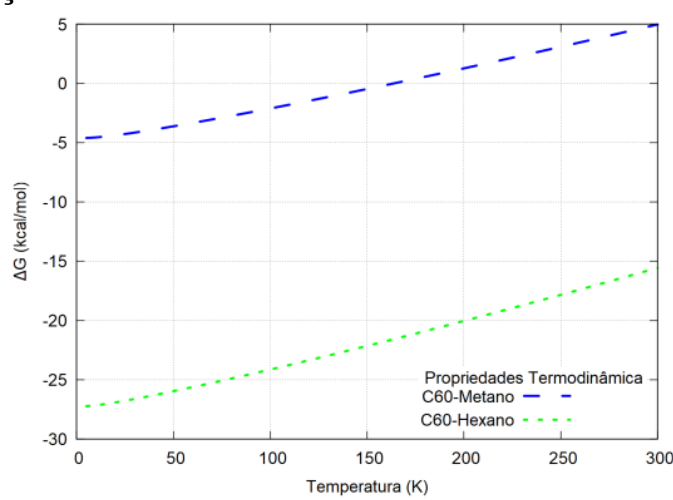

Figura 4. Variação da energia livre de Gibbs para o sistema C60-metano e C60-hexano

A Figura 4 apresenta a variação da energia livre de Gibbs, através dela nota-se que $\Delta \mathrm{G}$ é majoritariamente influenciada por $\Delta \mathrm{H}$.

\section{Conclusões}

Os resultados para os ajustes das CEPs se mostram satisfatórios, uma vez que os erros para ajuste ponto a ponto foram próximo de zero. Através das propriedades termodinâmicas obtidas torna-se possível observar o comportamento do fulereno C60 em solventes orgânicos. Para a continuação do trabalho, pretende-se relacionar as propriedades termodinâmicas com a solubilidade.

\section{Agradecimentos}

Á Coordenação de Aperfeiçoamento de Pessoal de Nível Superior (CAPES). Ao Centro de Computação de Alto Desempenho da Universidade Estadual de Goiás.

\section{Referências}

1. Kroto, H. W., Heath, J. R., O’Brien, S. C., Curl, R. F. \& Smalley, R. E. C. Nature 318, 162 (1985).

2. Goodarzi, S., Da Ros, T., Conde, J., Sefat, F. \& Mozafari, M. Fullerene. Mater. Today 00, (2017).

3. Neto, J. J. S. \& Costa, L. S.. Brazilian J. Phys. 28, 1-11 (1998).

4. Dunham, J. L. Physical Review 41, 721-731 (1932).

5. Baggio, A. R., Machado, D. F. S., Carvalho-Silva, V. H., Paterno, L. G. \& de Oliveira, H. C. B. Rovibrational spectroscopic constants of the interaction between ammonia and metallophthalocyanines: a theoretical protocol for ammonia sensor design. Phys. Chem. Chem. Phys. 19, 10843-10853 (2017). 